\title{
Analogy as a catalyst for cumulative cultural evolution
}

${ }^{1}$ Brand, C.O., ${ }^{1}$ Mesoudi, A., \& ${ }^{2}$ Smaldino, P. E.

${ }^{1}$ Human Behaviour and Cultural Evolution Group, College of Life \& Environmental Sciences, University of Exeter, Penryn, U.K.

${ }^{2}$ Department of Cognitive and Information Sciences, University of California, Merced, CA, USA

\begin{abstract}
Analogies, broadly defined, map novel concepts onto familiar concepts, making them essential for perception, reasoning, and communication. We argue that analogy-building served a critical role in the evolution of cumulative culture, by allowing humans to learn and transmit complex behavioural sequences that would otherwise be too cognitively demanding or opaque to acquire. The emergence of a protolanguage consisting of simple labels would have provided early humans with the cognitive tools to build explicit analogies and to communicate them to others. This focus on analogy-building can shed new light on the coevolution of cognition and culture, and addresses recent calls for better integration of the field of cultural evolution with cognitive science.
\end{abstract}

Keywords: analogy-building, language, protolanguage, cognition, culture, evolution 


\section{Cultural Evolution and Cognitive Science: Bridging the Gap}

Humans, like many other animals, have rich cultural lives in which behavioural variation is extensively acquired via social learning (see Glossary) from conspecifics, rather than via genetic inheritance (Hoppitt \& Laland, 2013; Thornton \& Clutton-Brock, 2011; Whiten et al., 1999). Yet unlike other species, humans have become one of the most dominant ecological forces on the planet, thriving in diverse ecological niches in a short space of evolutionary time (Hill et al., 2009). This ecological domination is often attributed to our uniquely cumulative culture, wherein our tools, technologies and customs 'ratchet-up' in complexity in a way that is yet to be unequivocally demonstrated in other species (Henrich, 2015; Mesoudi \& Thornton, 2018; Tennie et al., 2009; Tomasello, 1999). Why only humans exhibit cumulative culture is argued by many cultural evolutionists to be due to our high-fidelity social learning, prosociality, and/or teaching capacity (Boyd et al., 2011; Dean et al., 2012; Laland, 2018; Mesoudi, 2016). In contrast, cognitive scientists have argued that our capacity for higher-order abstraction is key to explaining the apparent discontinuity between human and non-human minds (Penn et al., 2008), and that we exceed other species in our ability to combine relations into higher order structures (Gentner \& Christie, 2008).

We believe that there is merit to both these perspectives, and that language and analogy crucially underpin both. Language is often acknowledged as essential for cumulative culture due to its role in teaching (Laland, 2017; Mesoudi, 2011), but it is still unclear what it is about language that differentiates mere culture from cumulative cultural evolution. We argue that language uniquely allows humans to make explicit analogies that aid the social transmission of complex new behaviours. (Boyd et al., 2011; Henrich, 2015; Laland, 2018; Morgan, 2016; Muthukrishna et al., 2018; Tomasello, 1999).

Analogy has been studied by cognitive scientists and linguists for decades, and a substantial corpus of work has demonstrated the importance of analogies for perception and categorisation (French, 1995; Hofstadter \& Sander, 2013; Mitchell, 1993), relational reasoning and abstract representation (Doumas et al., 2008; Gentner \& Goldin-Meadow, 2003a; Goldwater et al., 2018; Hummel \& Holyoak, 1997; Levinson, 2003), learning and reasoning in children (Navarrete \& Dartnell, 2017; Richland et al., 2006; Richland \& Begolli, 2016), and language evolution (Gentner, 2016; Goldwater, 2017; Goldwater \& Gentner, 2015), as well as the role of language in relational reasoning (Gentner \& Christie, 2008; Gentner \& Goldin-Meadow, 2003b; Levinson, 2003; Loewenstein \& Gentner, 2005; Lupyan, 2008) and in structuring our thinking (Gentner et al., 2001; Levinson, 2003; Lupyan \& Bergen, 2016; Lupyan \& Zettersten, 2020). Indeed, some argue that analogy is the 'core of our cognition' (Hofstadter, 2001; Hofstadter \& Sander, 2013). To the extent that cognition is central to cumulative culture (Heyes, 2018, 2019; Morin, 2016; Nettle, 2020; Scott-Phillips et al., 2018, Sperber, 2006), we suggest that analogy should therefore be considered core to cultural evolution too. Our aim here is not to review the aforementioned cognitive science literature in detail, but to point to where we think it is relevant to-and can contribute tocultural evolution research, which has been criticised for placing too much focus on high fidelity cultural transmission and various social learning strategies, and not enough on the cognitive processes or communication mechanisms that support cultural transmission 
(Heyes, 2016, 2019; Morin, 2016; Scott-Phillips et al., 2018; Sperber, 2006). A focus on analogy-building can remedy this, and help explain our species' unique capacity for cumulative culture.

\section{What is analogy-building?}

An analogy is a cognitive device by which a concept is mapped onto another concept based on its perceptual or conceptual similarities. We define analogy-building specifically as labelling novel perceptual or conceptual information on the basis of its relationship to some other previously perceived or conceived category (French, 1995; Hummel \& Holyoak, 1997). Although in everyday parlance analogies are often viewed as sophisticated linguistic flourishes, we use a broader definition of analogy that does not require complex language, claiming analogy to be a foundational aspect of perception, learning, and categorisation (Kallens et al., 2018; French, 2002; Gentner et al., 2001; Hofstadter \& Sander, 2013; Mitchell, 1993; Mitchell \& Hofstadter, 1990). While cognitive scientists typically focus on the computational aspects of producing or parsing analogies, we focus instead on the act of analogy-building to learn and, crucially, to communicate new information. A simple example is instructing a child to "cup" their hands (hold them in the shape of a cup) to hold water by saying the single word "cup", rather than dictating the precise arrangement that their fingers and hands must make. A more complex example is the story used to teach how to tie a bowline knot by referring to a rabbit running around a tree (Figure 1). Here, the end of the rope is labelled a "rabbit" and the loop a "burrow," to aid memorisation of the sequence. In this way, complex cultural information can be rapidly and effectively communicated.

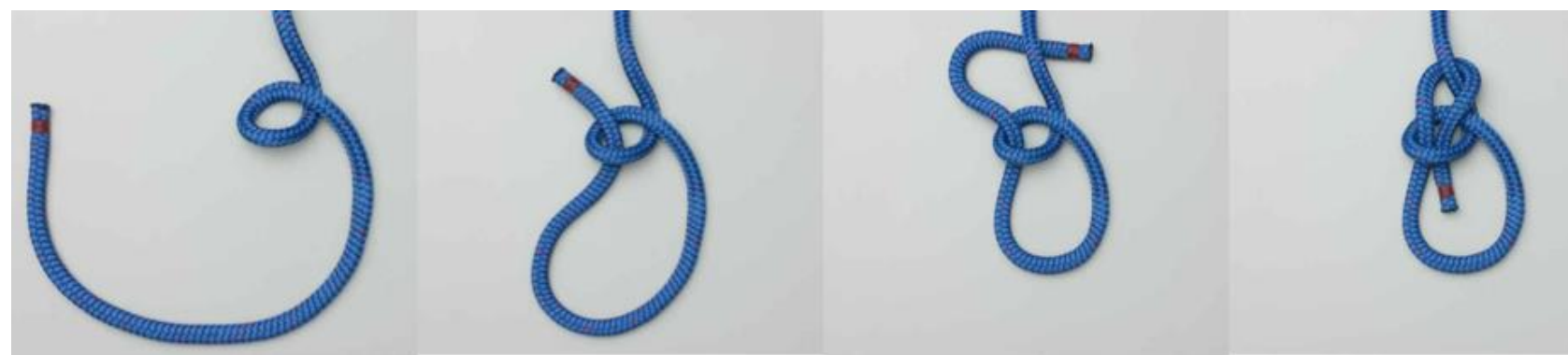

Figure 1: Images for tying a Bowline knot (adapted from www.animatedknots.com). Tying a Bowline contains multiple steps that must be performed in a specific order that is difficult to infer from the finished product. A commonly used 'rabbit' analogy aids memorisation of the order. (1) Here is the rabbit burrow. (2) The rabbit comes out of the burrow. (3) The rabbit goes around the tree. (4) The rabbit goes back down the burrow. This is typically much easier to remember than "put the rope end through the loop from underneath, wrap it around the other piece of rope above the loop, then put the same rope end through the same loop from above." Instead the information gets compressed into a story about a rabbit, which is more easily remembered due to its familiarity. Memory load is reduced to imagining that the rope end represents a rabbit, and the rest follows without having to recall any further information about the rope itself. These analogies are created based on shared perceptual features (e.g. a loop of rope looks like a rabbit burrow). 


\section{The role of analogy in language evolution}

We argue that the emergence of a simple protolanguage in human evolutionary history greatly enhanced our analogy-building capability compared to that of non-human animals, catalysing the process of cumulative cultural evolution in our lineage (Figure 2, Key Figure). Although some analogical reasoning can be performed in the absence of language (see Box 1), a form of protolanguage capable of simple verbal labels would have allowed early humans to link novel stimuli to familiar stimuli via analogical labels and to communicate this link to others (Stage 3 of Figure 2). As highlighted in Figure 2, we are predominantly concerned with a stage of language evolution in which word labels are present, but abstract words such as 'similar/different' need not be. Although comparison terms are clearly important in analogy-making (Özçalışkan et al., 2009), our interest is in the more general property of all words to act as analogies (Hofstadter \& Sander, 2013). Iconicity, i.e. a similarity between a sign and its meaning, is likely to have played a crucial role in the origin and evolution of words specifically, perhaps at Stage 2 of Figure 2 (Nielsen \& Dingemanse, 2020; Tamariz et al., 2018). Metaphor, meanwhile, likely plays a major role in shaping cognition (Lakoff \& Johnson, 2008; Thibodeau et al., 2019), but at a later evolutionary stage given that metaphor, as it is often conceived, requires complex linguistic conventions that are unlikely to have been present in protolanguage. Finally, we acknowledge that the process of cumulative cultural evolution contributes to the cultural evolution of language itself (Kirby et al., 2008). However, our focus here is on the origin of communicative analogy-building and its role in the evolution of human behaviour.

Although there is considerable debate as to when the first protolanguages emerged and whether they were predominantly lexical, gestural, musical or holistic, our theory fits with recent evidence that a protolanguage capable of providing verbal labels likely emerged sometime between Homo habilis and Homo sapiens, with Homo erectus a likely candidate (Bickerton, 2007, 2009; Fitch, 2010, 2017; Jackendoff \& Jackendoff, 2002). This timing is consistent with evidence suggesting that the manufacture of sophisticated stone tools likely relied on some form of protolanguage, with Oldowan tools potentially providing a selective gradient for the evolution of verbal teaching in early Homo (Morgan et al., 2015), and Acheulean tools almost certainly requiring a form of verbal protolanguage (Fitch, 2017). The social learning of tool use and production is often touted as a key selection pressure for the evolution of language and teaching (Kolodny \& Edelman, 2018; Laland, 2017). Evidence for a "cognitive coupling" theory of language evolution linking tool use and protolanguage comes from the observed coupling of the neural communication system with circuits related to complex motor sequence processing and action planning (Kolodny \& Edelman, 2018). We agree that the capacity for language likely coevolved with cognitive mechanisms for sequence processing in the context of tool production, and suggest that this coupling occurred as a result of analogy-based protolanguage scaffolding the learning of complex sequences and motor actions by chunking ordered sequences into compressed, more memorable, and thus more communicable information (see 'Ordered Sequence Learning' below). 


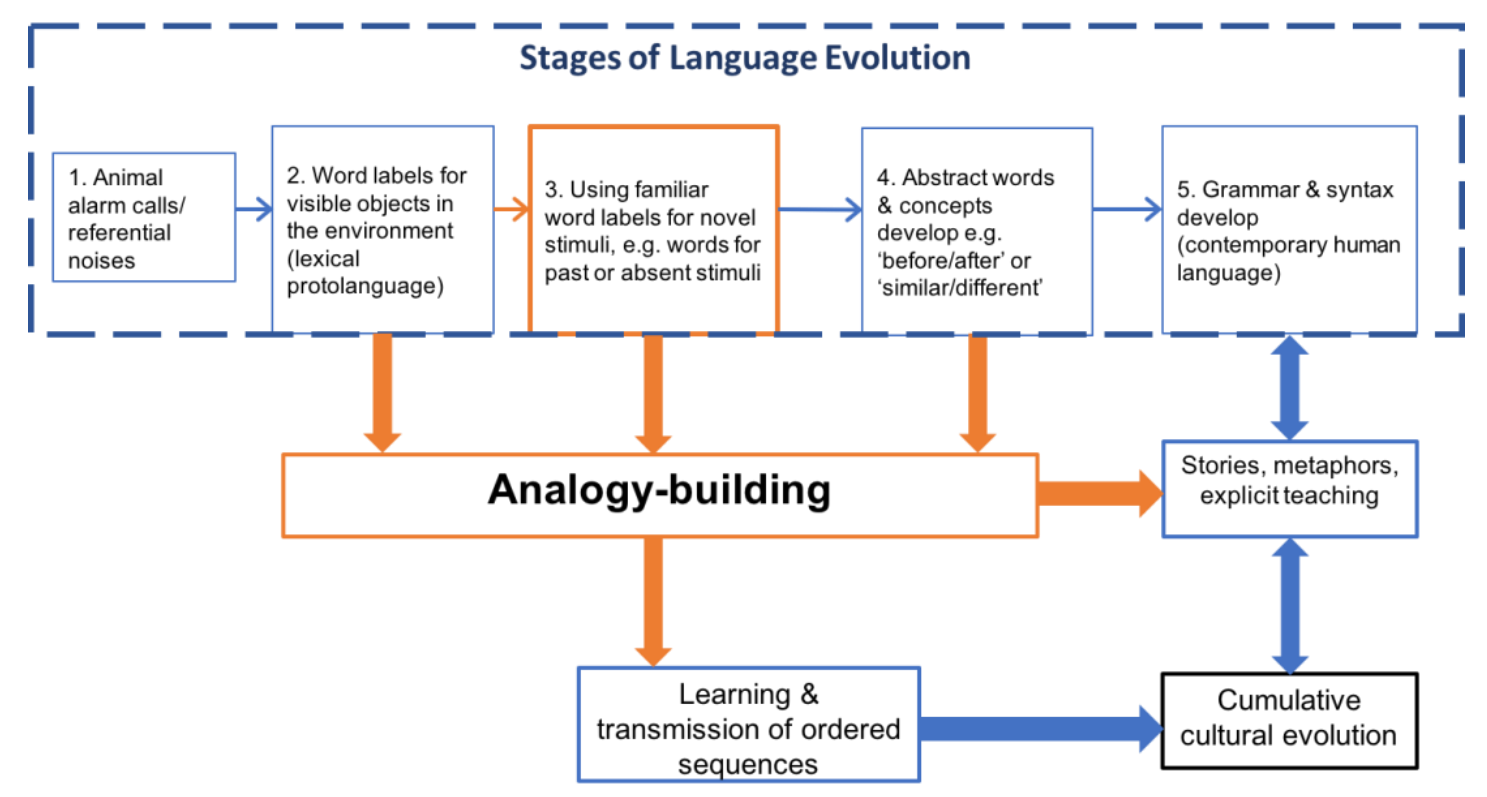

Figure 2: Proposed stages of protolanguage leading to analogy-building and cumulative cultural evolution. The thin arrows represent a step in language evolution, and the thick arrows represent a contribution to a process. The orange arrows represent the particular steps and processes that we are focussing on in this paper, and that we believe bring important insights to the study of cultural evolution. A form of protolanguage in which familiar words are used to label novel stimuli would allow for analogy-building (Stage 3), for example by labelling a new object or action as a past/absent object or action (e.g. labelling a new tool as a 'tooth' due to its functional and perceptual similarities: sharp, pointed, used to cut through meat). Being able to label and link multiple actions via an analogy would enhance the learning and transmission of ordered sequences, necessary for the cumulative cultural evolution of complex technology. Once human language evolved to its contemporary form (including complex grammar and syntax), then stories, metaphors and explicit teaching contribute to the process of cumulative cultural evolution as well, a process which feeds back into the cultural evolution of stories, metaphors and language itself, represented by the bidirectional arrows. We suggest that iconicity is likely to have been involved in Stage 2, and that metaphor would not be possible until Stage 5. It is Stage 3 that we are focussing on in this paper.

Our theory of analogy-building resolves a common critique of lexical protolanguage: how vocal learning could have arisen to provide the flexible use of words in the first place (Fitch, 2010). The need to vocally learn many words would be drastically reduced if analogybuilding is the core mechanism, because analogies reduce the number of words or phonemes that are needed to be under flexible control. If an individual only has five distinct sounds to their repertoire, these five sounds can be mapped onto many more than five stimuli using perceptual or conceptual similarities (Isbilen \& Christiansen, 2020). Thus, when using an already learnt utterance to refer to a novel stimulus, learning a novel utterance is not necessary. For example, imagine a prehistoric group with a lexical protolanguage including a word for "tooth" but no words for knife/tool. An individual from this group might call a novel, sharp and pointed item a "tooth" when first encountering it, with no need to vocally learn a new label. Eventually these sounds can be combined, shortened, or moderated to provide distinct utterances once new categories are further developed and established in the population. For example, "tooth" may drift into "toother" or "otooth" to distinguish reference to the tooth-like tool, or eventually "hand tooth." This hypothetical example demonstrates that if utterances for "hand" and "tooth" are already present in the 
protolanguage, analogy-building allows for more communicative flexibility without any increase in vocal flexibility per se.

\section{Box 1: Is Analogy-Building Unique to Humans?}

Although the explicit analogy-building we refer to here relies on a basic protolanguage, a more fundamental capacity for analogical reasoning likely exists in many non-human animals, at least in the sense that non-human animals are capable of categorisation (Bovet \& Vauclair, 1998; Delius, 1992; Range et al., 2008).

Analogical reasoning can be defined as applying learnt rules from one context to another. The extent to which non-human animals can perform analogical-reasoning tasks is debated. Evidence of analogical reasoning in rats was provided by Murphy and colleagues (Murphy et al., 2008), in an experiment in which rats learnt to differentiate sequences of stimuli based on simple rules, and then applied these learnt rules to novel stimuli sequences. However, a carefully controlled version of this experiment found that neither rats nor pigeons could generalise based on learnt rules, but that rats and pigeons instead appeared to generalize based on perceptual features(Maes et al., 2015). For example, when learning that stimuli combination " $A B$ " predicts a different outcome to either stimulus "A" or "B", humans were able to infer that "CD" predicted a different outcome to "C" and "D". In contrast, rats and pigeons incorrectly predicted the same outcome for the compound stimulus based on the perceptual overlap of $C D$ with its component parts $C$ and $D$.

The best evidence so far of analogical reasoning in non-human animals comes from relationalmatch-to-sample tasks in language- or symbol-trained apes (Gillan et al., 1981; Premack, 1983; Thompson et al., 1997; Thompson \& Oden, 2000). These experiments involve matching sequences of stimuli as described above. The likely difficulty that non-human animals have with such tasks is overcoming memory constraints, particularly for ordered sequences of stimuli (Ghirlanda et al., 2017). One solution to the problem of memory constraints is to chunk information by providing a shorthand for subsections of sequences. Arbitrary symbols allow such chunking, which could explain why those best able to perform these tasks are symbol- or language-trained apes.

Although non-human animals may be capable of analogical reasoning, evidence of their analogy use in communication is almost non-existent. Tantalising examples come from the languagetrained parrot Alex (Pepperberg, 1981), and a sign-language trained chimpanzee Washoe (Gardner et al., 1989), in which the animals combined learned words to describe a novel stimulus, such as "water bird" for "swan". Evidence so far suggests a non-human animal "can make its analogical knowledge explicit only if it is first provided with a symbol system by which propositional representations can be encoded and manipulated,"(Thompson \& Oden, 2000). This reliance on a symbol system supports our theory that a form of lexical protolanguage would have enabled early humans to explicitly build analogies between concepts, and crucially to communicate these with others. 


\section{Analogies aid the acquisition of complex information}

\section{Ordered sequence learning}

Analogies help individuals learn complex ordered sequences, which is a core requirement of cumulative cultural evolution. For example, tool-making often involves many cognitively opaque steps that must be followed in a specific order to successfully construct even the 'simplest' of tools such as handaxes. Language enables us to chunk information into relevant analogies, by which ordered sequences can be more efficiently learnt and transmitted. This fits evidence that sequence learning represents a key difference between humans and other species (Lupyan \& Casasanto, 2015; Lupyan \& Lewis, 2019; Perry \& Lupyan, 2017). A review of 108 experiments on sequence discrimination in 14 bird and mammal species found that non-human animals display a limited capacity for discriminating arbitrary sequences of stimuli, with some species needing thousands of trials to learn to discriminate a sequence of just two stimuli (Ghirlanda et al., 2017). In contrast, humans typically learn arbitrary sequences of 5-10 stimuli when presented just once or twice (Ghirlanda et al., 2017). Non-human animals' difficulty in discriminating ordered sequences is likely related to memory constraints, and language may help humans to overcome these constraints. Further evidence comes from a comparative study finding that human children are able to learn arbitrary spatial and item-based sequences through observation, whereas orangutans could only learn these via individual trial-and-error learning (Renner et al., 2020).

Analogy-building helps to explain this difference between human and non-human sequence discrimination. Recall the example of tying a bowline knot (Figure 1). Looking at the finished knot does not reveal how it was tied; the process involves multiple steps that must be performed in a specific order that is difficult to infer from the finished product. $A$ commonly used analogy or "story" aids memorisation of the order: "The rabbit comes out of the burrow, goes around the tree, and goes back down into the burrow." This is easier to remember than "put the rope end through the loop from underneath, wrap it around the other piece of rope above the loop, then put the same rope end through the same loop from above." The story about the rabbit uses familiar elements and schemas that are easily encoded-going in and out of burrows is typical rabbit behaviour. Moreover, it does not require encoding new information about either ropes or knots, as the analogy can be recalled and applied each time the knot is tied. Thus, the constraint of memory is overcome by chunking sections of the sequence into a shorthand via analogy. Shared cultural information like that of rabbits and burrows is crucial for communicating relevant analogies that can be understood by others, as discussed below.

\section{Labels, Categorisation and Conceptualisation}

Analogies aid in the categorization of novel objects and concepts (Gentner \& Christie, 2008; Loewenstein \& Gentner, 2005; Lupyan \& Casasanto, 2015; Lupyan \& Lewis, 2019; Perry \& Lupyan, 2017). Indeed, some scholars have taken the strong position that the act of 
categorisation is primarily an exercise in analogy-building (Mitchell 1993; French 1995; Hofstadter \& Sander 2013; Hofstadter 2001). Even without taking this strong stance, it is clear that much of our ability to perform cognitive computations stems from the compression of information into categories, and this is further aided by the ability to explicitly assign a categorical label to an object (Goldwater et al., 2018; Lupyan, 2008; Lupyan \& Casasanto, 2015; Perry \& Lupyan, 2017). Experimental evidence suggests that the ability to assign even previously meaningless labels to novel objects aids categorization of, and memory for, those objects (Goldstone et al., 2001; Lupyan \& Casasanto, 2015; Lupyan \& Lewis, 2019; Perry \& Lupyan, 2017). When familiar words or utterances can be used as labels for novel stimuli based on perceptual or relational similarities, analogies can be drawn between the properties of the label's referent and properties of the novel stimulus, further aiding categorization and reducing memory load. Here analogy-building not only reduces the cognitive load of novel information by anchoring it to already learnt information, but takes an abstract category from inside the individual's mind and gives it an external, audible utterance that allows it to be manipulated and communicated.

Relating a novel action to a familiar concept makes the cultural transmission of sequences and behaviours considerably easier. If a group has a verbal label for "cup", then asking a child to "cup" their hands by simply using the word "cup" is more effective than accurately describing the arrangement and movement that their fingers must make to achieve the same function. The difficulty of the latter is exacerbated if precise words for those actions and movements are not yet present in the lexicon. Considerable work in cognitive science has demonstrated the utility of characterizing knowledge as a structured network of semantic and causal relationships, and analogies as mappings between the structural and perceptual relationships in different domains ( French, 2002; Goldwater \& Gentner, 2015; Hummel \& Holyoak, 1997; McClelland et al., 2010; Mitchell, 1993). Analogy-building allows categories to become canalized into abstract concepts that can be manipulated cognitively, but, crucially, it also allows those abstract concepts to be communicated effectively. Manipulation underlies all reasoning, whether about technical or social information (Kallens et al., 2018; Hofstadter \& Sander, 2013). The ability to manipulate concepts both individually and communicatively leads to explicit causal reasoning and eventually to technical understanding.

\section{Causal reasoning and understanding}

The ability to build and communicate analogies underpins causal understanding of complex concepts and technologies, which in turn facilitates the cumulative cultural evolution of those concepts and technologies. Analogy-building is a form of categorisation, and as categories are used more regularly they become canalised into readily-accessible labels. Those labels can be used to build and communicate analogies to others, allowing communities to converge on folk theories of processes based on their similarities to other already-understood processes. These folk theories may be over simplistic by scientific standards, while still allowing for practical application. An example is the complex process used to make Samurai swords in pre-industrial times (Inoue, 2010; Martin, 2000). A rich 
understanding of the physico-chemical processes involved in making the swords both strong and sharp was not achieved until the $20^{\text {th }}$ century, and indeed the physics and chemistry underpinning the process continues to be updated. Instead, samurai sword making was surrounded by mythology and folk beliefs (Sinclaire, 2001). These beliefs allowed for high fidelity transmission of complex procedures based on analogies to other shared concepts and stories. Causal explanations that rely on analogies, even flawed analogies, are still causal explanations.

It is sometimes suggested that many products of cumulative cultural evolution can be successfully learnt and transmitted without causal understanding of how they are produced or why they are effective, and that causal reasoning or understanding often occurs post hoc (Henrich, 2015). However, causal understanding is not binary; rather, groups are constantly updating their theories of how systems work. Causal understanding and reasoning about a process may be scaffolded by (often flawed) analogies during learning and transmission. Although many anecdotal and ethnographic examples have been offered (Henrich, 2015), and experiments show that improvements in some tasks can occur in the absence of causal understanding (Derex et al., 2019), no experimental investigation to date has demonstrated that complex, opaque technology can cumulatively evolve in the absence of causal understanding. In one recent study (Derex et al., 2019), participants found increasingly efficient solutions to a task in which weights had to be placed on a wheel to make it descend a ramp as quickly as possible. Performance improved equally when causal theories could be transmitted alongside wheel designs as when designs were transmitted without causal theories. However, this task did not involve an ordered sequence of causally opaque steps, perhaps limiting the potential of causal understanding to improve solutions. Moreover, participants typically displayed some level of causal understanding, rather than none at all. In fact, a small number of participants spontaneously used analogies in their written accounts of the task, even though this was not intended to convey understanding to others. Participants compared the wheel task to ice-skating, Formula 1 driving, and being on a swing (for example: "The wheel covers the distance faster when the weights are close to the centre, just as if you stretch your legs on a swing that is spinning, it slows down," and "One could compare the wheel to a Formula 1 car, which needs a low centre of gravity" (Derex, pers comm 2020). We hypothesise that individuals rely on folk theories for the majority of systems we interact with day to day, and that these folk theories are underpinned by analogy.

\section{Analogies aid the communication of complex information}

\section{Communication \& teaching}

Human cumulative cultural evolution likely relies on teaching to ensure sufficiently high fidelity cultural transmission (Laland, 2017; Lucas et al., 2020). Some definitions of teaching require that the teacher adjusts their behaviour based on what the student does or does not know, leading to debates around whether theory of mind (i.e. understanding another's knowledge or belief state) is necessary for effective teaching (Michelle Ann Kline, 2015). Analogy-based teaching involves describing the elements of the new domain by 
analogy to another previously-learned domain, and aids learning in children (Lola \& Tzetzis, 2021; Vendetti et al., 2015). The evolutionary burden of theory of mind in the context of teaching is reduced with a protolanguage that can reference already-shared concepts via analogy. Referencing similarities to shared concepts automatically creates a shared perspective between teacher and student that does not require the ability to explicitly represent another's mental state. What is required is shared developmental and cultural information between the teacher and learner. As such, intense sociality is a prerequisite for analogy-based protolanguage.

For example, imagine a group who frequently hunt a "Natoo" bird with a large, fan-shaped crest (like a cockatoo). This group has a hand gesture that represents the Natoo, holding four fingers splayed with thumb tucked-in. Now imagine this same hand position is required for a novel action sequence, because a strand of rope must be weaved between the splayed fingers whilst held in position by the thumb. A teacher could use the "Natoo" utterance to instruct a student to hold their fingers splayed in such a way. This utterance would not be helpful to a member of another group who does not know the bird or does not associate the "Natoo" utterance with this gesture. The use of the "Natoo" utterance does not require an explicit theory of mind, only the knowledge that using this utterance is likely to cause an individual who hears it to use the gesture. In this way, analogy-based protolanguage creates a stepping stone between instances of teaching without explicit theory of mind (A. Thornton, 2006) and a teaching system relying on fully-fledged theory of mind like those present in contemporary human societies.

\section{Are analogies too WEIRD?}

It is sometimes claimed that teaching in pre-industrialised, small-scale societies does not take the explicit, verbal pedagogical form that it does in most WEIRD or post-industrialised societies (Boyette \& Hewlett, 2018). If explicit verbal teaching is only an artefact of contemporary WEIRD societies, this would undermine our argument that label-based protolanguage and explicit analogies coevolved to support cumulative cultural evolution. However, recent reviews of teaching in hunter-gatherers find that, although direct verbal instruction is relatively rare, it frequently occurs in domains of complex ecological knowledge or subsistence skill (Boyette \& Hewlett, 2018; Garfield et al., 2016; Hewlett \& Roulette, 2016; Kline et al., 2013). Evidence from seafaring navigators using traditional techniques in the Marshall Islands finds that the islanders conceptualise different wave patterns as resembling various tools used in their culture (Genz et al., 2009). For example, one term used to describe a wave pattern is the same term as the pole that they traditionally use to harvest fruit from a breadfruit tree, with the $\mathrm{V}$-shaped fork at the end resembling the pattern of the waves. Another term literally means 'pit for bird fighting' and refers to the small cage traditionally used to capture birds, with the intersecting bars of the cage resembling the intersecting waves (Genz et al., 2009). This kind of analogical labelling, where a familiar term is used to label a complex pattern or concept based on perceptual similarities, is the exact type of communication that our theory predicts. A similar example of analogous labelling comes from the Pirahã, who famously do not have fixed colour terms in 
their language, but instead refer to items as "looking like blood" to designate red (Everett, 2005). This reflects a type of experience-based communication that analogy-building relies upon, and that we predict would have been present in a hypothetical protolanguage. Indeed, this type of reference-based terminology is common in the world's languages across sensory modalities, particularly for the smell modality (e.g. in English, "smells fishy/eggy")(Majid et al., 2018).

\section{Box 2: Story-telling}

Stories provide shared knowledge of a set of situations that are likely to be useful to people in those societies. Thus, they shape the analogies that people have available and hence the ways in which they characterize the world, similarly to how formal models can provide mental models for scientists (Nowak et al., 2011). Humans have been characterised as "The Storytelling Animal", with evidence put forward for the centrality of stories in human life as well as their universality in human societies (Gottschall, 2012). Shared stories function as a communal analogy pool, in that the whole community is guaranteed a set of shared parables, myths and legends to support teaching of not only practical but also moral, religious and spiritual information. Having these ready-made shared concepts means not all concepts have to come from direct experience, but can be communicated within the group with reference to the story. This transmission of valuable cultural knowledge via story-telling has been particularly well documented as part of Aboriginal "dreamtime" culture and is common to many small-scale societies all over the world (Boyette \& Hewlett, 2018; Garfield et al., 2016; Mountford, 2020).

In WEIRD societies, story-telling is becoming a popular strategy for science communication initiatives (Joubert et al., 2019). Evidence suggests that putting the usually abstract and unfamiliar process of scientific discovery in the context of a story makes the information more accessible, and digestible and memorable analogies are an acknowledged form of science communication (Finkler \& Leon, 2019; Martinez-Conde \& Macknik, 2017). Analogies themselves are abundant in the history of science, whether for describing the structure of an atom as planets orbiting the solar system, enzyme receptor systems as a lock and key, or carbon dioxide as a glass pane in a greenhouse. Studies have found that metaphors and analogies are helpful for transmitting complex scientific concepts, whether for specific concepts such as thermodynamics and algorithms (Forišek \& Steinová, 2012; Haglund \& Jeppsson, 2012), or for science education in general (Aubusson et al., 2006; Kipnis, 2005). The use of metaphor and story-telling requires contemporary language, including some form of grammar, syntax, and recursion, and thus involves a more complex type of analogy-building than we are focussing on in this paper. Nevertheless, evidence suggests the ongoing use of analogies and metaphors today facilitates the ongoing cumulative cultural evolution of complex concepts and technology.

Although analogy-building per se has not been explicitly studied in small-scale societies, story-telling in some form appears to be universal in human communication and often accompanies complex cultural behaviours (see Box 2). For example, sand drawing in Vanuatu, in which intricate sequences of geometric shapes and symbols are traced in sand without lifting a finger from the ground, is often accompanied by a verbal story or song that communicates valuable cultural knowledge to others (Zagala, 2004). With over 300 different designs, the patterns seem too complex to learn via imitation alone, and although this has not been formally tested, it is reasonable to assume that story-telling or singing aids the 
learning of the sequence. Further evidence from contemporary foraging groups suggests that analogy is commonly used for identifying safe versus poisonous mushrooms, for example by describing certain mushrooms as wearing hats or stockings (Kaaronen, 2020). These examples are consistent with our theory that verbal analogy use is necessary for communicating information that has a heavy memory load or involves learning a large number of complex patterns or associations.

\section{Concluding remarks and future directions}

\section{All analogies are wrong, but some are cumulative}

Our theory of the role of analogy in the evolution of human cumulative culture is based on a consideration of the problem of communicating new information to naïve individuals. Analogies allow individuals to effectively communicate while avoiding the memory requirements of new jargon, enabling the linking of steps in an ordered sequence, and anchoring new information onto already-learnt concepts for easier encoding and future retrieval. As individuals become more familiar with a concept, it becomes more solidified in memory, and the scaffolding of the analogy can be shed or updated. Furthermore, once one becomes a specialist, relevant information is learnt, condensed, and chunked differently, allowing one to build qualitatively different analogies that link different-or differently organized-aspects of the system (Hofstadter, 2001). This is how jargon emerges in communities of experts. Experts no longer need the original anchors to remember or process information in their domain of expertise, and may find that the originally-learned analogies miss complexities and details that have since been discovered or understood. In these cases, an analogy learned early on may become a hindrance, an oversimplification that misses nuance. This may explain why many experts, scientists included, are often poor communicators in their domain of expertise-they are unwilling to oversimplify the nuances of their work for the goal of easing a novice into a new topic.

Analogies create value by highlighting similarities between two or more objects, events, or systems, similarities that exist in some particular contextual framing. A teddy bear is like a real bear by virtue of its shape and appearance, but this analogy does not provide us with useful information about the teddy bear's interior or whether we should approach a real bear for a cuddle. No analogy is perfect, because the whole point of an analogy is to draw connections between different things. Analogies often rely on complex linguistic structuring and will inevitably break down as more details of a system come to light. The limitations of analogies impinge on the history of science, as models of phenomena are replaced with new models that serve as increasingly generalizable analogies, what Kuhn (Kuhn, 1962) called 'paradigm shifts'. Examples of widespread analogies in the history of science include the characterization of light as a wave or of the brain as a computer. These analogies, once learned, become fodder for future analogies. For example, if the brain is a computer, then perhaps a society is a supercomputer (Smaldino \& Richerson, 2013). This accumulation of analogies is part of what facilitates cumulative culture. In the 1930s, when Alan Turing's original papers on computability were written, it would have been near impossible to explain 
to someone what a digital computer is and how it works; the underlying mechanics and mathematics were too dense to be easily accessible or understood. Now, after years of analogy to ease our familiarity ("desktops", "folders", "documents"), we have useful concepts about how computers work, and so we have a new analogy for the brain, an analogy that wouldn't have been possible a century ago and one that provides new ways to conceptualise parts of the brain and how it works.

\section{Analogy-building as the core of Cumulative Cultural Evolution?}

Hofstadter argues that analogies are the core of cognition (Hofstadter, 2001). We argue further that analogy-building was a catalyst for cumulative cultural evolution. Our hypothesis is that once a protolanguage consisting of simple labels evolved, our capacity for analogybuilding snowballed, allowing for the acquisition and transmission of complex ordered sequences that are the bedrock of cumulative cultural evolution. Although theories of cumulative cultural evolution provide a plausible explanation of human ecological success (Henrich, 2015; Laland, 2018), they have so far left individual cognition in a black box. These theories therefore provide little satisfactory explanation for the underlying computational and neurological underpinnings of human cultural transmission, nor our subjective cognitive experiences. Furthermore, our seemingly unique capacity for language has often been left in the wings, hailed as an effective sidekick for high-fidelity transmission, rather than being allowed centre stage in the story of how cultural innovations accumulate. Our focus on analogy-building shows why language should be in the limelight. As the roles of cognition and language are taken more seriously, insights from both cognitive science and cultural evolution can recombine to produce an improved understanding of the coevolution of cognition and culture.

Building on the ideas put forward in this paper, we propose a research programme in which the role of analogy in cumulative cultural evolution is investigated (see "Outstanding Questions"). Firstly, it should be possible to test using formal modelling when the benefits of an analogy-based communicative system can off-set some of the biological costs of developing a high-fidelity teaching mechanism that is needed for cumulative culture. We also predict that experimental transmission chains in which analogical labels (e.g. familiar labels from a different context) are used would allow for better transmission of novel behavioural sequences than chains which use non-analogical labels (e.g. nonsense words lacking any context). This type of experiment would differentiate between the effects of labelling with and without analogy-building. We further predict that the transmission of complex or counter-intuitive concepts are better transmitted via analogy than through purely literal argumentation. We hypothesise that participants in teaching conditions of cumulative culture transmission chain experiments (e.g. (Lucas et al., 2020; Morgan et al., 2015)) who use analogical language will better transmit the technology at hand than those who use nonanalogical language. These experimental set-ups could be expanded to include conditions that allow for analogical language, or simple analogical labelling of apparatus, to investigate if, when and how analogy-making improves the transmission of novel behavioural sequences or novel concepts. 
To summarise our argument, analogy-building allows an individual to learn a novel behaviour or concept by anchoring it to a familiar behaviour or concept. Once an individual could externalise this mapping as a verbal label, the analogy could be communicated to others, facilitating the spread of novel behaviours and concepts through communities, and ultimately catalysing cumulative cultural evolution.

\section{Glossary}

Analogy: A cognitive device by which a stimulus/object/action/concept is labelled or described based on its similarities to a familiar stimulus/object/action/concept. An example is the rabbit analogy used for tying a knot in Figure 1.

Analogy-building: Labelling a novel object or action in relation to its perceptual or conceptual similarities to a familiar object or action.

Analogical reasoning: The ability to make inferences about a new situation based on its similarity to an old situation, or the ability to transfer rules learnt in one domain to a new domain.

Categorisation: The compressing and classifying of perceptual information into discrete kinds on the basis of its relationship or similarity to previously perceived information.

Causal understanding: Knowledge of the constituents, properties, and physics of a system that allows an individual to explain or predict how antecedent circumstances lead to consequences. May be represented as a story or model.

Culture: Information capable of affecting individuals' behavior that is acquired from conspecifics through teaching, imitation, and other forms of social learning / cultural transmission. This information may be stored in individual brains or in the physical or institutional features that structure individuals' behaviors.

Cultural Evolution: The processes by which socially learned cultural variation changes over time; a theoretical framework developed from analogies between genetic and cultural change.

Cumulative Culture: Behaviours or artefacts that have accumulated innovations over time that increase their functionality or efficiency

Cumulative Cultural Evolution: The processes by which a culturally evolving behaviour or artefact accumulates innovations over time that increase its functionality or efficiency.

Protolanguage: A precursor to modern human language that consists of a more basic form of language, perhaps lacking grammar, syntax or recursion.

Social learning: Acquiring new behaviour through observation of, or interaction with, conspecifics. 
WEIRD: An acronym designating Western, Educated, Industrialised, Rich and Democratic societies. Often used to highlight the overreliance of psychological research on participants from these societies, which do not represent the majority of human variation.

\section{References}

Aubusson, P. J., Harrison, A. G., \& Ritchie, S. M. (2006). Metaphor and Analogy in Science Education. Springer Science $\&$ Business Media.

Bickerton, D. (2007). Language evolution: A brief guide for linguists. Lingua, 117(3), 510-526. https://doi.org/10.1016/j.lingua.2005.02.006

Bickerton, D. (2009). Adam's Tongue: How Humans Made Language, How Language Made Humans. Farrar, Straus and Giroux.

Bovet, D., \& Vauclair, J. (1998). Functional Categorization of Objects and of Their Pictures in Baboons (Papio anubis). Learning and Motivation, 29(3), 309-322. https://doi.org/10.1006/lmot.1998.1009

Boyd, R., Richerson, P. J., \& Henrich, J. (2011). The cultural niche: Why social learning is essential for human adaptation. Proceedings of the National Academy of Sciences, 108(Supplement 2), 10918-10925. https://doi.org/10.1073/pnas.1100290108

Boyette, A. H., \& Hewlett, B. S. (2018). Teaching in Hunter-Gatherers. Review of Philosophy and Psychology, 9(4), 771797. https://doi.org/10.1007/s13164-017-0347-2

Byrne, R. W., \& Whiten, A. (1990). Machiavellian Intelligence: Social Expertise and the Evolution of Intellect in Monkeys, Apes, and Humans. Behavior and Philosophy, 18(1), 73-75.

Contreras Kallens, P. A., Dale, R., \& Smaldino, P. E. (2018). Cultural evolution of categorization. Cognitive Systems Research, 52, 765-774. https://doi.org/10.1016/j.cogsys.2018.08.026

Dean, L. G., Kendal, R. L., Schapiro, S. J., Thierry, B., \& Laland, K. N. (2012). Identification of the Social and Cognitive Processes Underlying Human Cumulative Culture. Science, 335(6072), 1114-1118. https://doi.org/10.1126/science.1213969

Delius, J. D. (1992). Categorical discrimination of objects and pictures by pigeons. Animal Learning \& Behavior, 20(3), 301-311. https://doi.org/10.3758/BF03213385

Derex, M., Bonnefon, J.-F., Boyd, R., \& Mesoudi, A. (2019). Causal understanding is not necessary for the improvement of culturally evolving technology. Nature Human Behaviour, 3(5), 446-452. https://doi.org/10.1038/s41562019-0567-9

Doumas, L. A. A., Hummel, J. E., \& Sandhofer, C. M. (2008). A theory of the discovery and predication of relational concepts. Psychological Review, 115(1), 1-43. https://doi.org/10.1037/0033-295X.115.1.1

Dunbar, R. I. M. (1998). The social brain hypothesis. Evolutionary Anthropology: Issues, News, and Reviews, 6(5), 178190. https://doi.org/10.1002/(SICI)1520-6505(1998)6:5<178::AID-EVAN5>3.0.CO;2-8

Everett, D. L. (2005). Biology and language: A consideration of alternatives Stephen R. Anderson \& David W. Lightfoot, The language organ: linguistics as cognitive physiology. Cambridge: Cambridge University Press, 2002. Pp. xix+263. T. Givón, Bio-linguistics: the Santa Barbara lectures. Amsterdam: John Benjamins Publishing Company, 2002. Pp. xviii+383. Journal of Linguistics, 41(1), 157-175. https://doi.org/10.1017/S0022226704003093

Finkler, W., \& Leon, B. (2019). The power of storytelling and video: A visual rhetoric for science communication. Journal of Science Communication, 18(5), A02. https://doi.org/10.22323/2.18050202

Fitch, W. T. (2010). The Evolution of Language. Cambridge University Press.

Fitch, W. T. (2017). Empirical approaches to the study of language evolution. Psychonomic Bulletin \& Review, 24(1), 333. https://doi.org/10.3758/s13423-017-1236-5

Forišek, M., \& Steinová, M. (2012). Metaphors and analogies for teaching algorithms. Proceedings of the 43rd ACM Technical Symposium on Computer Science Education - SIGCSE '12, 15. https://doi.org/10.1145/2157136.2157147

French, Robert M. (2002). The computational modeling of analogy-making. Trends in Cognitive Sciences, 6(5), 200-205. https://doi.org/10.1016/S1364-6613(02)01882-X

French, Robert Matthew. (1995). The Subtlety of Sameness: A Theory and Computer Model of Analogy-making. MIT Press.

Gardner, R. A., Gardner, B. T., \& Cantfort, T. E. V. (1989). Teaching Sign Language to Chimpanzees. SUNY Press.

Garfield, Z. H., Garfield, M. J., \& Hewlett, B. S. (2016). A Cross-Cultural Analysis of Hunter-Gatherer Social Learning. In H. Terashima \& B. S. Hewlett (Eds.), Social Learning and Innovation in Contemporary Hunter-Gatherers: Evolutionary and Ethnographic Perspectives (pp. 19-34). Springer Japan. https://doi.org/10.1007/978-4-43155997-9_2 
Gentner, D. (2016). Language as cognitive tool kit: How language supports relational thought. American Psychologist, 71(8), 650-657. https://doi.org/10.1037/amp0000082

Gentner, D., \& Christie, S. (2008). Relational language supports relational cognition in humans and apes. Behavioral and Brain Sciences, 31(2), 136-137. https://doi.org/10.1017/S0140525X08003622

Gentner, D., \& Goldin-Meadow, S. (2003a). Language in Mind: Advances in the Study of Language and Thought. MIT Press.

Gentner, D., \& Goldin-Meadow, S. (2003b). Language in Mind: Advances in the Study of Language and Thought. MIT Press.

Gentner, D., Holyoak, K. J., Holyoak, K. J., \& Kokinov, B. N. (2001). The Analogical Mind: Perspectives from Cognitive Science. MIT Press.

Genz, J., AUCAN, J., Merrifield, M., Finney, B., Joel, K., \& Kelen, A. (2009). Wave Navigation in The Marshall Islands: Comparing Indigenous and Western Scinetific Knowledge of the Ocean. Oceanography, 22(2), 234-245.

Ghirlanda, S., Lind, J., \& Enquist, M. (2017). Memory for stimulus sequences: A divide between humans and other animals? Royal Society Open Science, 4(6), 161011. https://doi.org/10.1098/rsos.161011

Gillan, D. J., Premack, D., \& Woodruff, G. (1981). Reasoning in the chimpanzee: I. Analogical reasoning. Journal of Experimental Psychology: Animal Behavior Processes, 7(1), 1-17. https://doi.org/10.1037/0097-7403.7.1.1

Goldstone, R. L., Lippa, Y., \& Shiffrin, R. M. (2001). Altering object representations through category learning. Cognition, 78(1), 27-43. https://doi.org/10.1016/S0010-0277(00)00099-8

Goldwater, M. B. (2017). Grammatical Constructions as Relational Categories. Topics in Cognitive Science, 9(3), 776799. https://doi.org/10.1111/tops.12272

Goldwater, M. B., Don, H. J., Krusche, M. J. F., \& Livesey, E. J. (2018). Relational discovery in category learning. Journal of Experimental Psychology: General, 147(1), 1-35. https://doi.org/10.1037/xge0000387

Goldwater, M. B., \& Gentner, D. (2015). On the acquisition of abstract knowledge: Structural alignment and explication in learning causal system categories. Cognition, 137, 137-153. https://doi.org/10.1016/j.cognition.2014.12.001

Gottschall, J. (2012). The Storytelling Animal: How Stories Make Us Human. Houghton Mifflin Harcourt.

Haglund, J., \& Jeppsson, F. (2012). Using self-generated analogies in teaching of thermodynamics. Journal of Research in Science Teaching, 49(7), 898-921. https://doi.org/10.1002/tea.21025

Henrich, J. (2015). The Secret of Our Success: How Culture Is Driving Human Evolution, Domesticating Our Species, and Making Us Smarter. Princeton University Press.

Hewlett, B. S., \& Roulette, C. J. (2016). Teaching in hunter-gatherer infancy. Royal Society Open Science, 3(1), 150403. https://doi.org/10.1098/rsos.150403

Heyes, C. (2016). Blackboxing: Social learning strategies and cultural evolution. Philosophical Transactions of the Royal Society B: Biological Sciences, 371(1693), 20150369. https://doi.org/10.1098/rstb.2015.0369

Heyes, C. (2018). Enquire within: Cultural evolution and cognitive science. Philosophical Transactions of the Royal Society B: Biological Sciences, 373(1743), 20170051. https://doi.org/10.1098/rstb.2017.0051

Heyes, C. (2019). Cognition blindness and cognitive gadgets. Behavioral and Brain Sciences, 42, e187. https://doi.org/10.1017/S0140525X19001158

Hill, K., Barton, M., \& Hurtado, A. M. (2009). The emergence of human uniqueness: Characters underlying behavioral modernity. Evolutionary Anthropology: Issues, News, and Reviews, 18(5), 187-200. https://doi.org/10.1002/evan.20224

Hofstadter, D. R. (2001). Analogy as the Core of Cognition. In The Analogical Mind (p. 499).

Hofstadter, D. R., \& Sander, E. (2013). Surfaces and essences: Analogy as the fuel and fire of thinking. Basic Books.

Hoppitt, W., \& Laland, K. N. (2013). Social Learning: An Introduction to Mechanisms, Methods, and Models. Princeton University Press.

Hummel, J. E., \& Holyoak, K. J. (1997). Distributed representations of structure: A theory of analogical access and mapping. Psychological Review, 104(3), 427-466. https://doi.org/10.1037/0033-295X.104.3.427

Inoue, T. (2010). Tatara and the Japanese sword: The science and technology. Acta Mechanica, 214(1), 17-30. https://doi.org/10.1007/s00707-010-0308-7

Isbilen, E. S., \& Christiansen, M. H. (2020). Chunk-Based Memory Constraints on the Cultural Evolution of Language. Topics in Cognitive Science, 12(2), 713-726. https://doi.org/10.1111/tops.12376

Jackendoff, R., \& Jackendoff, R. S. (2002). Foundations of Language: Brain, Meaning, Grammar, Evolution. Oxford University Press.

Joubert, M., Davis, L., \& Metcalfe, J. (2019). Storytelling: The soul of science communication. Journal of Science Communication, 18(5), E. https://doi.org/10.22323/2.18050501

Kaaronen, R. O. (2020). Mycological rationality: Heuristics, perception and decision-making in mushroom foraging. Judgement and Decision Making, 15(5), 630-647.

Kipnis, N. (2005). Scientific Analogies and Their Use in Teaching Science. Science \& Education, 14(3), $199-233$. https://doi.org/10.1007/s11191-004-6406-y

Kirby, S., Cornish, H., \& Smith, K. (2008). Cumulative cultural evolution in the laboratory: An experimental approach to the origins of structure in human language. Proceedings of the National Academy of Sciences, 105(31), 1068110686. https://doi.org/10.1073/pnas.0707835105 
Kline, Michelle A., Boyd, R., \& Henrich, J. (2013). Teaching and the Life History of Cultural Transmission in Fijian Villages. Human Nature, 24(4), 351-374. https://doi.org/10.1007/s12110-013-9180-1

Kline, Michelle Ann. (2015). How to learn about teaching: An evolutionary framework for the study of teaching behavior in humans and other animals. Behavioral and Brain Sciences, 38. https://doi.org/10.1017/S0140525X14000090

Kolodny, O., \& Edelman, S. (2018). The evolution of the capacity for language: The ecological context and adaptive value of a process of cognitive hijacking. Philosophical Transactions of the Royal Society B: Biological Sciences, 373(1743), 20170052. https://doi.org/10.1098/rstb.2017.0052

Kuhn, T. S. (1962). The Structure of Scientific Revolutions: 50th Anniversary Edition. University of Chicago Press.

Lakoff, G., \& Johnson, M. (2008). Metaphors We Live By. University of Chicago Press.

Laland, K. N. (2017). The origins of language in teaching. Psychonomic Bulletin \& Review, 24(1), $225-231$. https://doi.org/10.3758/s13423-016-1077-7

Laland, K. N. (2018). Darwin's Unfinished Symphony: How Culture Made the Human Mind. Princeton University Press.

Levinson, S. C. (2003). Language and mind: Let's get the issues straight! In Language in mind: Advances in the study of language and thought (pp. 25-46). MIT Press.

Loewenstein, J., \& Gentner, D. (2005). Relational language and the development of relational mapping. Cognitive Psychology, 50(4), 315-353. https://doi.org/10.1016/j.cogpsych.2004.09.004

Lola, A. C., \& Tzetzis, G. C. (2021). The effect of explicit, implicit and analogy instruction on decision making skill for novices, under stress. International Journal of Sport and Exercise Psychology, O(0), 1-21. https://doi.org/10.1080/1612197X.2021.1877325

Lucas, A. J., Kings, M., Whittle, D., Davey, E., Happé, F., Caldwell, C. A., \& Thornton, A. (2020). The value of teaching increases with tool complexity in cumulative cultural evolution. Proceedings of the Royal Society B: Biological Sciences, 287(1939), 20201885. https://doi.org/10.1098/rspb.2020.1885

Lupyan, G. (2008). Taking symbols for granted? Is the discontinuity between human and nonhuman minds the product of external symbol systems? Behavioral and Brain Sciences, 31(2), 140-141. https://doi.org/10.1017/S0140525X0800366X

Lupyan, G., \& Bergen, B. (2016). How Language Programs the Mind. Topics in Cognitive Science, 8(2), $408-424$. https://doi.org/10.1111/tops.12155

Lupyan, G., \& Casasanto, D. (2015). Meaningless words promote meaningful categorization. Language and Cognition, 7(02), 167-193. https://doi.org/10.1017/langcog.2014.21

Lupyan, G., \& Lewis, M. (2019). From words-as-mappings to words-as-cues: The role of language in semantic knowledge. Language, Cognition and Neuroscience, 34(10), 1319-1337. https://doi.org/10.1080/23273798.2017.1404114

Lupyan, G., \& Zettersten, M. (2020). Does vocabulary help structure the mind? PsyArXiv. https://doi.org/10.31234/osf.io/b74u9

Maes, E., De Filippo, G., Inkster, A. B., Lea, S. E. G., De Houwer, J., D’Hooge, R., Beckers, T., \& Wills, A. J. (2015). Feature- versus rule-based generalization in rats, pigeons and humans. Animal Cognition, 18(6), 1267-1284. https://doi.org/10.1007/s10071-015-0895-8

Majid, A., Roberts, S. G., Cilissen, L., Emmorey, K., Nicodemus, B., O’Grady, L., Woll, B., LeLan, B., de Sousa, H., Cansler, B. L., Shayan, S., de Vos, C., Senft, G., Enfield, N. J., Razak, R. A., Fedden, S., Tufvesson, S., Dingemanse, M., Ozturk, O., ... Levinson, S. C. (2018). Differential coding of perception in the world's languages. Proceedings of the National Academy of Sciences, 115(45), 11369-11376. https://doi.org/10.1073/pnas.1720419115

Martin, G. (2000). Stasis in complex artefacts. In Technological innovation as an evolutionary process (pp. 90-100). Cambridge University Press.

Martinez-Conde, S., \& Macknik, S. L. (2017). Opinion: Finding the plot in science storytelling in hopes of enhancing science communication. Proceedings of the National Academy of Sciences, 114(31), 8127-8129. https://doi.org/10.1073/pnas.1711790114

McClelland, J. L., Botvinick, M. M., Noelle, D. C., Plaut, D. C., Rogers, T. T., Seidenberg, M. S., \& Smith, L. B. (2010). Letting structure emerge: Connectionist and dynamical systems approaches to cognition. Trends in Cognitive Sciences, 14(8), 348-356. https://doi.org/10.1016/j.tics.2010.06.002

Mesoudi, A. (2011). Cultural Evolution: How Darwinian Theory Can Explain Human Culture and Synthesize the Social Sciences. University of Chicago Press.

Mesoudi, A. (2016). Cultural Evolution: A Review of Theory, Findings and Controversies. Evolutionary Biology, 43(4), 481-497. https://doi.org/10.1007/s11692-015-9320-0

Mesoudi Alex \& Thornton Alex. (2018). What is cumulative cultural evolution? Proceedings of the Royal Society B: Biological Sciences, 285(1880), 20180712. https://doi.org/10.1098/rspb.2018.0712

Mitchell, M. (1993). Analogy-making as perception: A computer model. MIT Press.

Mitchell, M., \& Hofstadter, D. R. (1990). The emergence of understanding in a computer model of concepts and analogymaking. Physica D: Nonlinear Phenomena, 42(1-3), 322-334.

Morgan, T. J. H., Uomini, N. T., Rendell, L. E., Chouinard-Thuly, L., Street, S. E., Lewis, H. M., Cross, C. P., Evans, C., Kearney, R., de la Torre, I., Whiten, A., \& Laland, K. N. (2015). Experimental evidence for the co-evolution of 
hominin tool-making teaching and language. Nature Communications, 6, 6029.

https://doi.org/10.1038/ncomms7029

Morgan, Thomas J. H. (2016). Testing the Cognitive and Cultural Niche Theories of Human Evolution. Current Anthropology, 57(3), 370-377. https://doi.org/10.1086/686531

Morin, O. (2016). How Traditions Live and Die. Oxford University Press.

Mountford, C. P. (2020). The Dreamtime: Australian Aboriginal Myths. ETT Imprint.

Murphy, R. A., Mondragón, E., \& Murphy, V. A. (2008). Rule Learning by Rats. Science, 319(5871), $1849-1851$. https://doi.org/10.1126/science.1151564

Muthukrishna, M., Doebeli, M., Chudek, M., \& Henrich, J. (2018). The Cultural Brain Hypothesis: How culture drives brain expansion, sociality, and life history. PLOS Computational Biology, 14(11), e1006504. https://doi.org/10.1371/journal.pcbi.1006504

Navarrete, J. A., \& Dartnell, P. (2017). Towards a category theory approach to analogy: Analyzing re-representation and acquisition of numerical knowledge. PLOS Computational Biology, 13(8), e1005683. https://doi.org/10.1371/journal.pcbi.1005683

Nettle, D. (2020). Selection, adaptation, inheritance and design in human culture: The view from the Price equation. Philosophical Transactions of the Royal Society B: Biological Sciences, 375(1797), 20190358. https://doi.org/10.1098/rstb.2019.0358

Nielsen, A. K., \& Dingemanse, M. (2020). Iconicity in Word Learning and Beyond: A Critical Review. Language and Speech, 0023830920914339. https://doi.org/10.1177/0023830920914339

Nowak, A., Rychwalska, A., \& Borkowski, W. (2011). Why Simulate? To Develop a Mental Model. Journal of Artificial Societies and Social Simulation, 16(3), 12.

Özçalışkan, Ş., Goldin-Meadow, S., Gentner, D., \& Mylander, C. (2009). Does language about similarity play a role in fostering similarity comparison in children? Cognition, 112(2), 217-228. https://doi.org/10.1016/j.cognition.2009.05.010

Penn, D. C., Holyoak, K. J., \& Povinelli, D. J. (2008). Darwin's mistake: Explaining the discontinuity between human and nonhuman minds. Behavioral and Brain Sciences, 31(2), 109-130. https://doi.org/10.1017/S0140525X08003543

Pepperberg, I. M. (1981). Functional Vocalizations by an African Grey Parrot (Psittacus erithacus). Zeitschrift Für Tierpsychologie, 55(2), 139-160. https://doi.org/10.1111/j.1439-0310.1981.tb01265.x

Perry, L. K., \& Lupyan, G. (2017). Clarifying the label-categorisation link. Language, Cognition and Neuroscience, 32(8), 950-953. https://doi.org/10.1080/23273798.2017.1328124

Premack, D. and P. (1983). The Mind of an Ape. Eweb:44780. https://repository.library.georgetown.edu/handle/10822/796601

Range, F., Aust, U., Steurer, M., \& Huber, L. (2008). Visual categorization of natural stimuli by domestic dogs. Animal Cognition, 11(2), 339-347. https://doi.org/10.1007/s10071-007-0123-2

Renner, E., Patterson, E. M., \& Subiaul, F. (2020). Specialization in the vicarious learning of novel arbitrary sequences in humans but not orangutans. Philosophical Transactions of the Royal Society B: Biological Sciences, 375(1805), 20190442. https://doi.org/10.1098/rstb.2019.0442

Richland, Lindsey E., Morrison, R. G., \& Holyoak, K. J. (2006). Children's development of analogical reasoning: Insights from scene analogy problems. Journal of Experimental Child Psychology, 94(3), 249-273. https://doi.org/10.1016/j.jecp.2006.02.002

Richland, Lindsey Engle, \& Begolli, K. N. (2016). Analogy and Higher Order Thinking: Learning Mathematics as an Example. Policy Insights from the Behavioral and Brain Sciences, 3(2), 160-168. https://doi.org/10.1177/2372732216629795

Scott-Phillips, T., Blancke, S., \& Heintz, C. (2018). Four misunderstandings about cultural attraction. Evolutionary Anthropology: Issues, News, and Reviews, 27(4), 162-173. https://doi.org/10.1002/evan.21716

Sinclaire, C. (2001). Samurai: The Weapons and Spirit of the Japanese Warrior. Lyons Press.

Smaldino, P. E., \& Richerson, P. J. (2013). Human Cumulative Cultural Evolution as a Form of Distributed Computation. In P. Michelucci (Ed.), Handbook of Human Computation (pp. 979-992). Springer New York. https://doi.org/10.1007/978-1-4614-8806-4_76

Sperber, D. (2006). Why a deep understanding of cultural evolution is incompatible with shallow psychology. In The Roots of Human Sociality. Berg Publishers.

https://www.researchgate.net/profile/Dan_Sperber/publication/30527615_Why_a_deep_understanding_of_cultu ral_evolution_is_incompatible_with_shallow_psychology/links/0fcfd50c649dfb9600000000/Why-a-deepunderstanding-of-cultural-evolution-is-incompatible-with-shallow-psychology.pdf

Tamariz, M., Roberts, S. G., Martínez, J. I., \& Santiago, J. (2018). The Interactive Origin of Iconicity. Cognitive Science, 42(1), 334-349. https://doi.org/10.1111/cogs.12497

Tennie, C., Call, J., \& Tomasello, M. (2009). Ratcheting up the ratchet: On the evolution of cumulative culture. Philosophical Transactions of the Royal Society B: Biological Sciences, 364(1528), 2405-2415. https://doi.org/10.1098/rstb.2009.0052

Thibodeau, P. H., Matlock, T., \& Flusberg, S. J. (2019). The role of metaphor in communication and thought. Language and Linguistics Compass, 13(5), e12327. https://doi.org/10.1111/lnc3.12327 
Thompson, R. K., Oden, D. L., \& Boysen, S. T. (1997). Language-naive chimpanzees (Pan troglodytes) judge relations between relations in a conceptual matching-to-sample task. Journal of Experimental Psychology. Animal Behavior Processes, 23(1), 31-43. https://doi.org/10.1037//0097-7403.23.1.31

Thompson, R. K. R., \& Oden, D. L. (2000). Categorical Perception and Conceptual Judgments by Nonhuman Primates: The Paleological Monkey and the Analogical Ape. Cognitive Science, 24(3), 363-396. https://doi.org/10.1207/s15516709cog2403_2

Thornton, A. (2006). Teaching in Wild Meerkats. Science, 313(5784), 227-229. https://doi.org/10.1126/science.1128727

Thornton, Alex, \& Clutton-Brock, T. (2011). Social learning and the development of individual and group behaviour in mammal societies. Philosophical Transactions of the Royal Society B: Biological Sciences, 366(1567), 978-987. https://doi.org/10.1098/rstb.2010.0312

Tomasello, M. (1999). The Human Adaptation for Culture. Annual Review of Anthropology, 2, 509-529.

Tomasello, M., \& Call, J. (1997). Primate Cognition. Oxford University Press.

Vendetti, M. S., Matlen, B. J., Richland, L. E., \& Bunge, S. A. (2015). Analogical Reasoning in the Classroom: Insights From Cognitive Science. Mind, Brain, and Education, 9(2), 100-106. https://doi.org/10.1111/mbe.12080

Whiten, A., Goodall, J., McGrew, W. C., Nishida, T., Reynolds, V., Sugiyama, Y., Tutin, C. E. G., Wrangham, R. W., \& Boesch, C. (1999). Cultures in chimpanzees. Nature, 399(6737), 682-685. https://doi.org/10.1038/21415

Zagala, S. (2004). Vanuatu Sand Drawing. Museum International, 56(1-2), 32-35. https://doi.org/10.1111/j.13500775.2004.00455.x 